\title{
Un outil politique : le voyage présidentiel en province
}

Deux visites du président Mitterrand dans le Finistère $(1981,1985)$

\section{Patrick Gourlay}

\section{(2) OpenEdition \\ Journals}

Édition électronique

URL : http://journals.openedition.org/abpo/1043

DOI : $10.4000 / a b p o .1043$

ISBN : 978-2-7535-1500-0

ISSN : 2108-6443

Éditeur

Presses universitaires de Rennes

Édition imprimée

Date de publication : 20 décembre 2005

Pagination : 85-115

ISBN : 978-2-7535-0243-7

ISSN : 0399-0826

Référence électronique

Patrick Gourlay, «Un outil politique : le voyage présidentiel en province », Annales de Bretagne et des Pays de l'Ouest [En ligne], 112-4 | 2005, mis en ligne le 20 décembre 2007, consulté le 02 mai 2019. URL : http://journals.openedition.org/abpo/1043; DOI : 10.4000/abpo.1043 


\title{
Un outil politique : le voyage présidentiel en province

\author{
Deux visites du président Mitterrand \\ dans le Finistère $(1981,1985)$
}

\author{
Patrick GOURLAY \\ Professeur d'histoire-géographie au collège de Lanmeur \\ Doctorant, Université de Bretagne Occidentale
}

Tout déplacement présidentiel est depuis toujours un mode d'exercice du pouvoir. Cette "propagande par la vue " s'appuie sur une mise en scène mobilisant les marqueurs républicains, le protocole et les notables mais elle se veut aussi l'occasion d'une rencontre avec la population. Le Président vient se montrer et joue en alternance sur deux registres opposés : la proximité et le maintien à distance du peuple. Avec la Ve République, cela se renforce et la théâtralisation du pouvoir reste très protocolaire. Cette pratique politique du voyage de souveraineté, utilisée par tous les Présidents de la République, contient en elle même des ressources dont le chef de l'État use avec différents objectifs.

François Mitterrand a utilisé ses voyages pour façonner son image présidentielle et pour agir dans le jeu politique. La visite présidentielle mitterrandienne est alors, plus que jamais, un outil politique qui s'appuie à la fois sur son parcours personnel et sur le protocole républicain. En 1981, dans un contexte marqué par l'alternance, il cherche à rassurer sur la capacité de la gauche à gouverner et à s'inscrire dans la durée. En 1985, à quelques mois des législatives, il tente de bâtir son personnage de "Président rassembleur " dans une Bretagne qui reste encore une terre d'opposition.

\section{Pourquoi sortir de l'Élysée?}

\section{Le Président « a vu de ses yeux}

\section{au moins quinze millions de Français" (de Gaulle)}

De tout temps, les chefs de l'État ont parcouru la France afin de marquer la maîtrise de leur territoire. L'inventeur des voyages fut Sadi Carnot qui en fit soixante-douze entre 1887 et 1894. Il s'agissait alors pour lui de 
" républicaniser la nation " et " d'affermir la fonction présidentielle ${ }^{1}$ ». Les Présidents réinvestissent alors la pratique ancienne du déplacement du monarque en province. Cet héritage de l'Ancien Régime consistait à mettre en relation tous les points du pays; la France était conçue comme un grand puzzle que le monarque se devait de reconstituer par ses voyages. La visite présidentielle comme recherche de légitimation populaire est une donnée permanente ancrée dans le temps long. Lorsque le Président voyage ne s'agit-il pas pour lui de parcourir pour unir et de chercher ainsi à maîtriser le territoire national? Ce déplacement, du centre vers la périphérie, n'estil pas aussi la marque de la volonté présidentielle de faire descendre la République au plus profond du territoire?

Trois mois avant l'élection présidentielle de 1965, le Général de Gaulle tient une conférence de presse à l'Élysée lors de laquelle il définit le rôle du Président de la République et l'importance du contact direct qu'il doit maintenir avec le peuple.

" Je ne crois pas que, jamais, de tels contacts aient été multipliés autant depuis sept ans. 30 allocutions adressées au pays tout entier par la voie de la radio et de la télévision; 12 conférences de presse intégralement diffusées; 36 discours solennellement prononcés lors de cérémonies publiques; séries de voyages accomplis - indépendamment d'au moins 200 apparitions officielles à Paris - dans les 94 départements de la métropole et d'outre-mer, au cours desquels le chef de l'État a vu, de ses yeux, au moins quinze millions de Français, invité à conférer avec lui tous les membres du Parlement, tous les Corps constitués, tous les conseillers généraux, tous les maires de France, visité environ 2500 communes, dont toutes les principales, répondu dans les Hôtels de ville à la bienvenue de près de 400 conseils municipaux et de 100000 notables, parlé depuis des estrades dans plus de 600 localités à la population assemblée, dialogué avec tant de personnes qu'on ne pourrait les compter et serré d'innombrables mains ${ }^{2}$."

Pour de Gaulle, le déplacement en province vise à maintenir un lien direct avec les citoyens. Voilà pourquoi, il lui apparaît essentiel que ce soit l'ensemble du pays qui soit ainsi visité par le chef de l'État. Cette vision est maintenue par ses successeurs qui, tous à leur manière, vont s'appliquer à entretenir ce rituel avec des objectifs généraux qui restent globalement les mêmes. Le Président souhaite sortir de l'Élysée pour sentir la France réelle. Pourquoi? Pour certains, la fonction le coupe des réalités. Son cabinet lui assure que tout est sous contrôle et maîtrisé. Or, comme le Président n'est pas dupe, il souhaite aller au contact des Français pour se faire une idée par lui-même de telle ou telle situation. C'est la raison pour laquelle, même si le programme lui laisse peu d'occasions de rencontrer la population, François Mitterrand n'hésite pas parfois à faire arrêter le cortège et à s'engager dans

1. SANSON, Rosemonde, " La République en représentation. À propos des voyages en province des Présidents de la République (1879-1914) ", dans La France démocratique, Paris, Publications de la Sorbonne, 1998, p. 372.

2. Conférence de presse de De Gaulle, citée dans "Allocutions et messages ", Mémoires d'espoir, 1999, Paris, Plon, p. 941-942. 
des bains de foule improvisés qui sont pour lui le moment de demander directement : "Dites-moi quels sont les problèmes ${ }^{3}$. " Le Président joue alors sur la proximité et crée un lien direct entre le chef de l'État et les citoyens.

Pour d'autres, comme Louis Le Pensec, cette vision des choses est erronée. Selon lui, il est au contraire, l'homme le mieux informé de France. Et s'il souhaite aller au contact des réalités, c'est pour d'autres raisons. Les facteurs qui déterminent les voyages sont toujours les mêmes, mais ils ont un coefficient d'importance qui varie selon le contexte du déplacement. Première motivation : il lui faut aller au contact des Français car le Président est toujours en campagne et il a en tête sa réélection. "Sans jamais le dire, le Président planifie sur la durée de son septennat sa rencontre avec les Français ${ }^{4}$. "Il s'agit ainsi pour lui de couvrir l'ensemble du territoire.

Les nécessités de politique intérieure constituent le deuxième facteur déterminant. Le Président profite d'une de ses sorties pour aider son Premier ministre et pour mettre en valeur l'action de son gouvernement. Ses petites phrases sont parfaitement bien choisies pour être relayées par les médias nationaux. À titre d'exemple, lors de sa venue en Bretagne, en octobre 1985, le Président Mitterrand manifeste clairement sa confiance à Fabius : "Je suis très content de ce gouvernement. Ce sont de bons ministres avec un très remarquable Premier Ministre, qui ont la confiance entière du Président de la République ${ }^{5}$."

Le déplacement est aussi l'occasion de lancer des ballons d'essais. Dans ce cas, le lieu doit fournir le contexte le meilleur et le plus porteur pour que la parole présidentielle puisse bénéficier des plus grandes retombées médiatiques. Comme le souligne Marylise Lebranchu, se déplacer, c'est aussi reconnaître l'action menée par des élus dans une ville, un département ou une région : "La visite présidentielle est un geste fort dont les gens comme les élus locaux ont besoin. Le talent du chef de l'État est donc de donner, par sa visite, de la fierté aux gens visités ${ }^{6}$. " L'ultime visite de François Mitterrand dans le Finistère en octobre 1994 entre dans ce cadre d'un déplacement qui se voulait, tout du long, un hommage au travail fourni par les municipalités socialistes de Brest et de Quimper. Liée souvent aussi à ce souhait de reconnaissance, il y a la volonté, de la part du Président, de légitimer un élu en cédant à ses pressions amicales. Bernard Poignant tenait beaucoup à ce que le Président Mitterrand vienne en visite officielle à Quimper durant son mandat de maire et il l'avait sollicité à plusieurs reprises. Il obtient satisfaction le 18 octobre 1994, quelques mois avant les municipales et la fin du mandat présidentiel.

3. Entretien avec Kofi Yamgnane, secrétaire d'État aux Affaires sociales et à l'Intégration (1991-1993), le 10 juillet 2003.

4. Entretien avec Louis Le Pensec, ministre de la Mer (1981-1983), ministre des DomTom (1988-1993), ministre de l'Agriculture et de la Pêche (1997-1998), le 23 octobre 2003.

5. Le Parisien libéré, 9 octobre 1985.

6. Entretien avec Marylise Lebranchu, secrétaire d'État aux PME (1997-2000), ministre de la Justice, Garde des Sceaux (2000-2002), le 6 juin 2003. 
Dernier facteur qui détermine la volonté de sortir de l'Élysée : c'est le besoin de décompresser. Selon Louis Le Pensec : "Ces voyages sont toujours appréciés par les Présidents. Il s'agit toujours de moments forts et l'accueil chaleureux qui leur est fait leur permet de se ressourcer ${ }^{7}$. "

Au total, les motivations sont variées mais elles ont un point commun : le déplacement présidentiel a toujours un sens politique et la mise en scène du pouvoir doit être l'occasion de mettre en valeur le chef de l'État. François Mitterrand s'est lui aussi inscrit dans cette longue tradition assimilée par la République depuis Sadi Carnot et utilisée à plein sous la Ve République.

Le Finistère a connu trente déplacements présidentiels entre 1874 et 2004. On compte sept visites sous la III ${ }^{\mathrm{e}}$ République et une de Vincent Auriol sous la IVe République. Tous les Présidents de la Ve République se sont rendus dans ce département au moins à une reprise. Avec vingt et une visites depuis 1958, on relève une nette augmentation de ces voyages. Cela s'explique naturellement par la révolution des transports mais cela témoigne aussi de l'importance occupée par la force de dissuasion nucléaire française et par les sites de Brest et de l'île Longue au sein de la Défense nationale. En classant ces visites, on peut ainsi relever, entre 1874 et 2004, onze visites à caractère militaire, neuf spécifiquement civiles et dix déplacements mixtes. Les visites militaires ajoutées aux déplacements mixtes confirment la sur-représentation militaire qui apparaît comme une singularité finistérienne ${ }^{8}$.

\section{Comment paraître?}

\section{Essai de typologie des visites présidentielles}

Tout déplacement du chef de l'État est politique. La conjoncture, le motif officiel de la visite, les circonstances, dictent la posture choisie par le Président de la République. On peut tenter d'en définir une typologie.

La visite en chef des Armées réaffirme le pouvoir régalien du Président. C'est sous cette posture que le Finistère l'accueille le plus souvent, depuis 1874, lorsqu'il vient pour le lancement de navires, le départ de la Jeanne d'Arc, l'inspection de l'École navale ou pour visiter les installations de la force de dissuasion nucléaire de l'île Longue.

Le déplacement présidentiel pour inaugurer des équipements publics exprime la reconnaissance de l'État pour le travail accompli par les élus. À travers cette présence présidentielle, il s'agit de donner une audience nationale à une réussite technologique. Gaston Doumergue vient, par

7. Entretien avec Louis Le Pensec, le 23 octobre 2003.

8. GouRLAY, Patrick, Les voyages des Présidents de la République dans le Finistère (18742004). La mise en scène du pouvoir présidentiel en province. Mémoire de DEA en histoire contemporaine, Université de Bretagne Occidentale, 2004. Thèse en cours, sous la direction de Christian Bougeard. 
exemple, en 1930, inaugurer le pont de Plougastel parce qu'il constitue " un nouveau record de l'industrie française " et que cet ouvrage est " unique au monde ${ }^{9}$ ". Mais certaines inaugurations ne sont que des prétextes donnés au Président pour venir saluer ses amis politiques. C'est le cas pour François Mitterrand qui préside l'inauguration du nouvel Hôtel de Ville de Concarneau en 1988 et celle de la faculté des Lettres de Brest Victor Segalen en 1994.

Le Président effectue des visites compassionnelles suite à une catastrophe. Il se présente alors en "Père de la nation ". Valéry Giscard d'Estaing effectue ce type de visite en août 1978 lors de la marée noire de l'Amoco Cadiz. François Mitterrand se déplace à Landéda en 1986 quelques jours après le naufrage d'un canot de la SNSM. Ces déplacements sont-ils à rapprocher de l'ancienne pratique des rois thaumaturges? Car si le Président ne guérit plus par un toucher réel, il peut néanmoins avoir un effet mobilisateur par sa venue et permettre d'accélérer la mobilisation des fonds et les services de l'État tout en rendant, au nom de la nation, un hommage appuyé aux victimes et aux sauveteurs.

Dernier type de visite : le grand voyage régional. C'est le type de déplacement le plus riche car il permet de se présenter sous plusieurs postures : chef de l'État, ami politique, Père de la nation, "Président rassembleur ". Il s'agit, en quelques jours, d'aller au contact de la population, de rencontrer des amis politiques, ou de croiser le fer avec des adversaires, et d'aller dans des lieux symboliques. À titre d'exemple, le Général de Gaulle, lors de son grand voyage de septembre 1960, se montre en chef des Armées lorsqu'il est à bord du Clemenceau mais redevient le chef politique lorsqu'il intervient sur l'Algérie dans son discours à Brest. Puis il réapparaît comme l'homme du 18 juin 1940 lors d'une cérémonie à l'île de Sein.

Les visites de François Mitterrand s'inscrivent dans cette typologie. Le Président Mitterrand adopte deux grands types de postures dans le Finistère : la visite en chef des Armées et le déplacement en chef de l'État. C'est une constante sur les deux septennats durant lesquels il fit au total sept déplacements :

- 24 juillet 1981 : visite de l'Île Longue et passage à la mairie de Brest;

- 25 mai 1985 : visite de l'île Longue pour la première campagne du SNLE L'Inflexible;

- 7 et 8 octobre 1985 : grand voyage en Bretagne;

- 22 août 1986 : visite compassionnelle après le naufrage d'un canot de la SNSM.

- 29 novembre 1988 : départ de la Jeanne d'Arc et inauguration de l'hôtel de Ville de Concarneau.

- 7 mai 1994 : déplacement à Brest pour la mise à l'eau du Charles-de-Gaulle.

- 18 octobre 1994 : déplacement à Quimper et à Brest.

9. L'Illustration, 30 août 1930. 


\section{1 : la visite du Président en chef des Armées}

\section{L'affirmation du pouvoir régalien}

L'analyse des quatre visites présidentielles en chef des Armées, fait apparaître que ces déplacements sont décidés " d'en haut ", négociés entre l'Élysée et l'État-Major de la Marine nationale avec l'influence du lobby de la dissuasion nucléaire. La venue en personne du Président vise à montrer à l'autorité militaire l'intérêt qu'il porte à ces questions. On peut dégager trois grandes motivations " internes ". Mitterrand vient à deux reprises réaffirmer que la dissuasion nucléaire reste la doctrine de notre défense (visites de 1981 et de 1985). Sa venue peut également viser à rassurer les marins à un moment où les restrictions budgétaires créent de l'inquiétude (visite de 1988). Enfin, le déplacement présidentiel est aussi un moment solennel pour fêter la mise à flot d'un fleuron de la Marine, (le Charles-de-Gaulle, en 1994). À ces objectifs liés à la politique interne de défense, on peut ajouter les motivations qui entrent dans le cadre des relations internationales. À travers ses déplacements, François Mitterrand envoie des messages au monde; messages relayés par la présence de journalistes français et étrangers. En 1981, il se presse de réaffirmer le maintien de la doctrine de dissuasion; et en 1985, il confirme lors de sa venue à Brest son refus de la "guerre des étoiles " souhaitée par les États-Unis.

Ces visites sont organisées, de bout en bout, par la Marine nationale et en particulier par le Préfet maritime. Le Président arrive dans le département par la base aéronavale de Landivisiau : l'accueil est assuré par les autorités militaires et on relève parfois la présence des élus locaux (maires des communes limitrophes de la base). Il s'envole ensuite en hélicoptère vers l'île Longue avant de se rendre sur le site militaire brestois et à la préfecture maritime. La Marine joue avec la fiction qu'elle accueille son chef des Armées dans un espace déterritorialisé; espace non brestois, purement militaire : un endroit " $\mathrm{X}$ ». Les élus brestois ne sont d'ailleurs pas systématiquement reçus par le chef de l'État. Par les moyens de transport utilisés, par les lieux visités et les gens rencontrés, la Marine entend faire une démonstration de force. Et elle ne souhaite pas partager cette venue avec le maire de Brest et la société civile. Il est par exemple hors de question que le Président reçoive en audience des représentants de syndicats agricoles. Il y a pourtant des exceptions à ces visites exclusivement militaires. Le président Mitterrand, qui n'entend pas se faire dicter sa conduite par l'armée, se rend en 1981 à l'Hôtel de ville de Brest et en 1988 à Concarneau.

On peut distinguer deux grands types de visites à la Marine. Il y a tout d'abord ce que l'on pourrait appeler des visites " ouvertes " : ces déplacements sont pleinement médiatisés, et sont l'occasion de délivrer un double message, aux marins et à la nation. Les visites de juillet 1981, de novembre 1988 et de mai 1994 entrent dans cette typologie. Il y a ensuite des visites "fermées" : il s'agit ici de reconnaître toute la place occupée par la Marine au sein de la Défense nationale. On pense à la visite de mai 1985. Et il est tout à fait probable, selon Pierre Maille, qu'à l'instar de 
la plongée incognito de Pierre Mauroy en novembre 1981, " le Président Mitterrand ait fait des visites, non annoncées, dans l'enceinte militaire et qu'il ait participé en secret à des exercices à bord de $\operatorname{SNLE}^{10}$ "; ce qui illustrerait le lien privilégié existant entre le Président et la Marine du fait de la place occupée par l'arme nucléaire.

La place occupée par la Marine nationale, dans les déplacements présidentiels dans le Finistère, révèle l'importance du site brestois et de l'Île Longue dans le dispositif de dissuasion nucléaire français. Tous les Présidents y sont venus en tant que chefs des Armées; même Alain Poher durant son intérim suite au décès de Georges Pompidou. L'article 15 de la Constitution de 1958 précise que : « Le Président de la République est le chef des Armées. Il préside les conseils et comités supérieurs de la défense nationale. " De plus, le décret du 14 janvier 1964, relatif aux forces aériennes stratégiques dit clairement que le Président " a seul qualité pour décider l'emploi du feu nucléaire ${ }^{11}$ ". Lorsque le Président vient à Brest, il endosse son habit militaire. Sa visite est alors soit " fermée ", c'est-à-dire uniquement destinée à la Marine, ou " ouverte " lorsqu'elle est le moyen d'adresser un message à la nation; message touchant aux forces armées. Enfin, elle peut être strictement militaire ou être mixte lorsqu'elle comporte aussi une visite et une rencontre des élus et de la population à Brest ou ailleurs dans le Finistère.

\section{Objectif de la première visite à Brest : rassurer la Marine}

Cette visite est la première effectuée par le Président Mitterrand dans le Finistère. Elle se déroule dans un contexte politique particulier, celui de l'alternance, et marque, par sa mixité (militaire et civile), la volonté d'imprimer une rupture de la part du nouveau Président. Depuis la passation de pouvoir, le 21 mai 1981, Mitterrand s'emploie à rassurer l'armée et lui envoie une série de signes. Le 27 mai, le Président se fait expliquer, dans les sous-sols de l'Élysée, le fonctionnement du PC Jupiter qui commande le feu nucléaire. Il peut de là mettre en action les trois composantes de l'arme nucléaire stratégique : terrestre avec les missiles du plateau d'Albion; aérienne avec les Mirages équipés de missiles; et maritime avec les cinq sous-marins lanceurs d'engins de la Force Océanique Stratégique (FOST). Puis le 11 juin, il rend visite au poste de commandement sous-terrain de Taverny.

Parmi les premiers actes du nouveau Président, il y a donc une tournée des sites militaires essentiels à la stratégie de défense française. Cela s'explique parce que l'alternance est vécue, ainsi que le rappelle Louis Le Pensec, comme un " choc dans les État-Majors ${ }^{12}$ ". Ceci malgré " la conversion progressive des partis de gauche et du centre à la stratégie de

10. Entretien avec Pierre Maille, maire de Brest (1982-1983)-(1989-2001), Président du Conseil Général du Finistère depuis 1998, le 13 août 2003.

11. ForCade, Olivier, DuHAMEl, Éric, VIal, Philippe (dir.), Militaires en République (18701962), Paris, Publications de la Sorbonne, 1999, p. 49.

12. Entretien avec Louis Le Pensec, le 23 octobre 2003. 
dissuasion nucléaire; elle mûrit de 1974 à 1978 et est acquise entre 1978 et $1981^{13}$ ». On ne peut en effet qu'être frappé, dans les premiers mois, par les efforts déployés par le Premier ministre et par le ministre de la Défense, Charles Hernu, pour rassurer. Ainsi, Pierre Mauroy adresse-t-il un hommage à ces hommes en soulignant leur attachement aux valeurs républicaines :

" [...] J'ai pu y sentir le civisme et le dévouement à la République du personnel de la FOST. [...] La sécurité de la France repose largement sur la compétence, la disponibilité et la patience attentive des sous-mariniers. Le gouvernement dont j'assume la direction en a pleine conscience ${ }^{14}$."

De son côté, l'État-Major, par l'intermédiaire de sa revue, Cols bleus, prend acte : "Ce premier contact de M. Mauroy avec les forces armées montre l'intérêt que porte le Premier ministre aux problèmes de défense et en particulier à la composante principale de la force nucléaire de dissuasion ${ }^{15}$. "Quelques mois plus tard, Pierre Mauroy fit une plongée de trente-six heures, incognito, à bord du sous-marin Le Tonant, à l'occasion de la centième patrouille de ce SNLE. C'est dans ce contexte particulier que le Président Mitterrand souhaite " aller voir un sous-marin nucléaire ${ }^{16}$ ". La gauche doit faire rapidement un geste fort à destination de la Marine. La visite à l'île Longue, le 24 juillet 1981, entre dans cette optique; mais elle recouvre également d'autres aspects plus politiques.

Avec cette première visite officielle aux Armées, le Président Mitterrand veut concrétiser sa fonction; montrer à tous que c'est désormais lui qui détient le pouvoir du feu nucléaire, ce " sceptre présidentiel ${ }^{17}$ ". Le rituel présidentiel est respecté à la lettre; c'est une manière de se montrer en chef suprême. À son arrivée, les honneurs militaires lui sont rendus. Puis, lorsqu'il monte à bord du Terrible l'équipage lui rend les honneurs au coup de sifflet traditionnel; sur le pont du sous-marin, la marque présidentielle est hissée. Il s'agit du drapeau tricolore avec en son centre les initiales «F.M » en lettres d'or. Sa venue, avec le ministre de la Défense, le chef d'ÉtatMajor de la Marine, le commandant de la FOST, le place aux yeux de tous comme le chef des Armées et le légitime dans sa fonction.

\section{Le protocole fait partie de la "pédagogie du changement »}

Le protocole permet de bien montrer que désormais c'est François Mitterrand qui détient le pouvoir. Il s'agit là de ce que Pierre Mauroy a appelé " la pédagogie du changement ${ }^{18}$ ". L'Élysée et Matignon souhaitent

13. GAUTIER, Louis, Mitterrand et son armée (1990-1995), Paris, Grasset, 1999, p. 18.

14. Cols bleus, 11-18 juillet 1981.

15. Ibidem.

16. FaVier, Pierre, Martin-Rolland, Michel, La décennie Mitterrand, tome 1, Paris, Le Seuil, 1990, p. 466.

17. Ibidem, p. 63.

18. Monclaire, Stéphane, "L'usage du protocole", dans LaCroix, Bernard, Lagroye, Jacques (dir.), Le Président de la République, Usages et genèse d'une institution, Paris, Presses de la Fondation Nationale de Sciences Politiques, 1992, p. 155. 
en effet que les règles protocolaires soient entièrement respectées en particulier lors des déplacements en province. Louis Le Pensec, alors ministre de la Mer, souligne que "Pierre Mauroy avait donné des consignes écrites très strictes à ce sujet ${ }^{19}$ ". Il s'agit ainsi de montrer à tous, marins, notables politiques et population, que le pouvoir a changé de main. Mais en même temps, c'est une époque où « le président semble tout aussi soucieux d'être l'incarnation d'une permanence. Il respecte scrupuleusement les institutions de la Ve République et les attributs de la fonction présidentielle. Dès son intronisation Mitterrand met en scène le président en majesté ${ }^{20}$ ". C'est donc un Président très soucieux du protocole qui se rend à Brest. Pour rassurer une Marine inquiète de l'arrivée aux responsabilités des socialistes, François Mitterrand s'emploie à jouer sur tous les éléments de continuité : utilisation des symboles, avec un rituel qui est respecté à la lettre, et posture alternant distance et proximité.

Si les destinataires du message sont les marins, c'est bien l'ensemble de la population qui est visée. Sa visite est médiatisée et il arrive accompagné de soixante-dix journalistes. Marque visible du changement relevée par Ouest-France le 25-26 juillet 1981 : la présence d'envoyés spéciaux de L'Humanité. Mais ce changement n'est qu'apparent car le chef des Armées fait tout, au contraire, pour souligner qu'il s'inscrit dans la continuité de la doctrine nucléaire du pays. "La France doit disposer de cette arme qui est l'instrument principal de sa stratégie de dissuasion... non pas pour la guerre, mais pour qu'il n'y ait pas de guerre ${ }^{21}$. " Lors de cette conférence de presse, il se ménage un effet d'annonce qui ne peut que satisfaire la Marine : "La France va construire un septième sous-marin nucléaire, et la décision devrait être prise rapidement pour qu'il puisse entrer en service vers $1990^{22}$. "C'est cette information qui est reprise à la " Une " des journaux. L'objectif semble atteint : le Président a montré son intérêt pour la Marine et a réaffirmé que la doctrine de défense de la France restait bien la dissuasion nucléaire.

En même temps qu'il adopte la posture distante du chef des Armées, Mitterrand souhaite établir avec les marins un rapport de proximité. Avec les officiers supérieurs, il déjeune dans le carré des officiers du Terrible. Plus marquant encore, il demande à ce que le commandant du sous-marin lui présente un à un les cent trente-cinq membres de l'équipage. Ce contact direct entre le Président et ces hommes n'a rien d'anecdotique car au moment où le sous-marin appareille, il déclare : "C'est pour moi un moment très fort. Quand on sait le poids de sa mission et puisqu'il y a contacts directs entre le Président de la République et ce sous-marin ${ }^{23}$. " En se ren-

19. Entretien avec Louis Le Pensec, le 23 octobre 2003.

20. ABELEs, Marc, "Symbolique présidentielle et culture républicaine ", dans AgUlHON, Maurice, Cultures et folklore républicains, Paris, éditions du CTHS, 1995, p. 215.

21. Ouest-France, 25-26 juillet 1981.

22. Le Télégramme, 25-26 juillet 1981.

23. Ouest-France, 25-26 juillet 1981. 
dant à l'île Longue, François Mitterrand est venu affirmer, par sa présence comme chef suprême des Armées, la continuité de la politique de défense. Il le dit clairement à tous : "Nous entendons rester, comme cela a été décidé il y a bien longtemps, maîtres de nos propres décisions lorsqu'il s'agit de la sauvegarde de notre territoire national ${ }^{24}$. " Ces précisions, sur la doctrine du nouveau Président, devaient sans doute être faites car " malgré certains efforts, l'arrivée de la gauche au pouvoir a constitué un séisme pour toute une partie de l'armée ${ }^{25}$ ". C'est sûrement aussi pour cela que Mitterrand " enfonce le clou " à Brest en rappelant que " les socialistes français sont pour la défense nationale c'est-à-dire pour l'indépendance nationale. Cela fait déjà quatre ans qu'ayant adopté cette stratégie nous sommes restés logiques avec nous-mêmes ${ }^{26}$ ".

Ce qui ressort de cette visite à l'île Longue, c'est moins la posture attendue de chef des Armées que la manière dont celui-ci utilise l'ensemble des moyens à sa disposition pour apparaître l'incarnation de la notion de continuité dans le domaine de la dissuasion. Cette volonté va jusqu'à faire visiter au Président Mitterrand le sous-marin sur lequel Valéry Giscard d'Estaing avait fait une plongée en novembre 1974. Simple hasard? En tous les cas, il est symbolique.

\section{Le Président rend visite à ses amis politiques brestois}

Le premier voyage présidentiel du septennat recensé est celui effectué en Lorraine en octobre $1981^{27}$. Cette visite brestoise n'est donc pas prise en compte sans doute parce qu'elle est à dominante militaire. Or, en se rendant à la mairie de Brest, ce 24 juillet 1981, il s'agit sans doute de la toute première visite officielle du Président Mitterrand à une municipalité dans le cadre de ses nouvelles fonctions. Venir à la mairie de Brest dans ce contexte d'une visite à la Marine est à placer sous le signe de la rupture avec les pratiques présidentielles antérieures. C'est aussi une façon de rendre hommage à ses amis politiques.

La rupture tout d'abord : contrairement à la pratique de Valéry Giscard d'Estaing, en 1974 et 1977, Mitterrand trouve qu'il est " important qu'un Président de la France ne boude pas Brest quand il vient à l'île Longue ${ }^{28}$ ". De plus, il montre ainsi à l'autorité militaire qu'on ne lui impose rien. Car la Marine nationale ne souhaite généralement pas partager la visite présidentielle. Elle ne veut pas que le déplacement du chef des Armées soit dilué en quelque sorte par d'autres rencontres.

24. Le Télégramme, 25-26 juillet 1981.

25. Entretien avec Louis Le Pensec, 23 octobre 2003.

26. Le Télégramme, 25-26 juillet 1981.

27. FAvier, Pierre, MarTin-Rolland, Michel, La décennie Mitterrand, tome 1, Paris, Le Seuil, 1990, p. 661.

28. Ouest-France, 25-26 juillet 1981. 
La proximité politique ensuite : rendre visite à la municipalité brestoise, c'est aussi marquer sa reconnaissance pour les socialistes de la cité du Ponant qui l'ont emporté au premier tour des municipales de mars 1977 avec trente-deux voix d'avance. "Ne pas venir n'aurait pas été compris " dit Pierre Maille alors premier adjoint au maire de Brest ${ }^{29}$, ville où François Mitterrand totalise 51,5\% des voix au second tour des présidentielles. François Cuillandre y voit lui une "forme de compensation au fait que Francis Le Blé, qui était un proche de Mitterrand, n'ait pas été désigné par les militants comme candidat du PS pour les législatives de juin $1981^{30}$ ". Pour les élus, il s'agit d'une visite symbolique de la nouvelle donne politique. D'ailleurs, le Président n'hésite pas à afficher ses amitiés : " J'étais à l'île Longue, Brest n'est pas loin. J'y suis venu parce que j'y ai des amis personnels. Je tenais à saluer la population; c'est pour cela que je suis venu voir ses représentants ${ }^{31}$ ". L'annonce de cette venue n'est pas faite auparavant; on peut donc la qualifier de visite "surprise " dans le sens où la population n'est pas mobilisée pour accueillir le Président.

Le maire présente les élus brestois puis dresse le portrait de sa ville. La discussion dure une demi-heure à huis clos. Le rituel de la remise du cadeau a lieu dès cette première rencontre : le maire de Brest remet au Président une estampe de Louis Garneray représentant la Penfeld en 1821. Voilà pour le don. Pour le contre-don, les élus UDB « sollicitent l'honneur d'être reçus par M. les ministres de l'Intérieur, de la Communication, de l'Énergie, et de l'Éducation nationale, afin de présenter les problèmes qui se posent à la Bretagne et les solutions que quinze années de travail militant au service des travailleurs bretons lui ont inspirées ${ }^{32}$ ". Ce rituel du don et contre-don trouve sa concrétisation dans une promesse présidentielle " Je reviendrai " et dans sa déclaration laconique reliant bien les deux moments de sa journée brestoise : "Les problèmes importants de la ville tiennent à des décisions d'État ${ }^{33}$. " Le chef de l'État montre ici aux élus qu'il prend en compte la spécificité de la situation de leur ville dont l'activité économique est intimement liée à la présence de la Marine nationale. C'est sans doute cela aussi le changement.

Au début de son mandat, le Président souhaite se montrer proche des gens. À la sortie de la mairie, les officiants (président, entourage, élus brestois), et les spectateurs se mêlent comme jamais dans un grand bain de foule. Il n'y a pas de barrières et malgré le moment de la visite (15 heures, en plein été), il y a 2000 personnes qui se sont massées pour saluer le Président. Les photographies publiées dans la presse montrent une grande proximité et un accès direct au chef de l'État; ce qui ne se reproduira jamais

29. Entretien avec Pierre Maille, le 13 août 2003.

30. Entretien avec François Cuillandre, maire de Brest depuis 2001, ancien secrétaire fédéral du PS dans le Finistère, le 18 mars 2005.

31. Le Télégramme, 25-26 juillet 1981.

32. Ouest-France, 25-26 juillet 1981.

33. Le Télégramme, 25-26 juillet 1981. 
de la sorte lors des visites ultérieures. Cela illustre sans doute l'atmosphère d'état de grâce qui flottait ce 24 juillet 1981 dans les rues de Brest la rose.

\section{Le Président et « Le double corps du roi »}

L'analyse du déroulement de cette journée de juillet 1981 permet de souligner que si le changement est à l'ordre du jour depuis mai, le Président Mitterrand semble très soucieux d'apparaître comme l'incarnation d'une continuité. C'est tout le contraire de l'approche qu'avait manifestée Valéry Giscard d'Estaing dès 1974. Celui-ci souhaitait se rapprocher des Français et moderniser le protocole afin de " lutter contre ce décalage entre l'État et la société, [quitte à] s'en prendre aux pesanteurs d'une certaine tradition républicaine ${ }^{34}$ ". Pour l'élu socialiste, il s'agit à l'inverse de s'appuyer sur cette tradition républicaine pour sculpter son image d'homme d'État et pour faire oublier qu'il fut l'opposant le plus marqué aux institutions de la Ve République. Il impose donc le respect du protocole, qui est le produit historique d'une longue évolution, il met en scène son pouvoir et adopte la posture du chef des Armées. Dès lors, il est assez tentant d'adapter la théorie des Deux Corps du roi à cette étude de cas brestoise.

"Le roi a en lui deux corps, c'est-à-dire un corps naturel et un corps politique. Son corps naturel, considéré en lui-même, est un corps mortel, sujet à toutes les infirmités qui surviennent par Nature ou Accident, à la faiblesse de l'enfance ou de la vieillesse, et aux déficiences semblables à celles qui arrivent aux corps naturels des autres gens. Mais son corps politique est un corps qui ne peut être vu ni touché, consistant en une société politique et en un gouvernement et constitué pour la direction du peuple et la gestion du bien public, et ce corps est entièrement dépourvu d'enfance, de vieillesse et de tous autres faiblesses et défauts naturels auxquels est exposé le corps naturel, et pour cette raison, ce qui fait le roi en son corps politique ne peut être invalidé ou annulé par une quelconque incapacité de son corps naturel ${ }^{35}$."

Le roi aurait donc la particularité de posséder deux natures : l'une mortelle; l'autre pérenne. Si l'on adapte ce concept au cas présidentiel, on peut considérer que le Président lui aussi possède un double corps : l'un est mortel, c'est le corps de l'homme engagé en politique; l'autre est l'incarnation du corps politique, de la France permanente. Lors de cette visite du 24 juillet 1981, François Mitterrand concentre les deux postures. Le chef des Armées marque la permanence de la doctrine militaire française : il se place alors dans le sillage de ses prédécesseurs depuis de Gaulle. Il incarne le corps politique qui a en charge le destin de la France; le corps immortel. Puis, avec ses amis socialistes, c'est l'homme politique, l'élu du peuple de gauche qui se déplace; c'est le Président pris dans le présent de l'action. Ainsi, cette visite, vue à travers la grille de lecture classique de E. Kantorowitz, permet-

34. ABELES, Marc, "François Mitterrand et la roche de Solutré ", dans CREPU, Michel (dir.), Hauts lieux, Autrement, série Mutations n 115, Paris, mai 1990, p. 134.

35. Kantorowicz, Ernest, Les Deux Corps du roi, Paris, Gallimard, 1989, p. 21-22. 
elle de souligner que, derrière le déplacement officiel en chef des Armées, c'est sans doute le souci d'incarner la continuité de l'État, au-delà de l'alternance de 1981, qui paraît être inscrit dans cette mise en représentation, à Brest, du nouveau Président de la République.

\section{François Mitterrand en "Président rassembleur "}

\section{Le grand voyage en Bretagne des 7 et 8 octobre 1985 : un Tro Breizh en terre d'opposition}

Ce voyage présidentiel, le douzième en province depuis 1981, est le dernier avant les législatives de 1986. Pour François Mitterrand, venir en Bretagne revêt donc une importance considérable. Il s'agit de tenter de séduire les électeurs bretons centristes dont les suffrages pourraient être déterminants en mars 1986 pour la gauche au plan national. Car la France électorale se compose de deux masses comparables qui votent à gauche et à droite : ce qui fait basculer la victoire vers l'un ou l'autre camp ce sont justement les suffrages centristes que l'on sait attirer à soi. En ce mois d'octobre 1985, le Président vient en Bretagne en terre d'opposition. Les rituels présidentiels mobilisés, associés aux rencontres protocolaires avec les élus (dans le Finistère, les maires rencontrés sont tous de droite, à l'exception de Morlaix et Guimaëc), et avec des visites d'entreprises, visent à renforcer sa légitimité.

Dans ce contexte, cette visite est donc un événement légitimant d'abord pour le chef de l'État. Ceci d'autant plus que le voyage présidentiel est marqué d'une part, par le contexte pré-électoral des législatives et la future cohabitation, et d'autre part, par l'opposition frontale organisée par le parti communiste. Car la cohabitation est déjà dans toutes les têtes. La presse nationale voit dans ce déplacement " un mode d'emploi de l'après $1986^{36}$ ". Et la visite à Quimper est annoncée comme une rencontre test car le maire, Marc Bécam, est décrit comme étant de " la race des cohabitants $^{37}$ ". L'opposition du parti communiste s'inscrit quant à elle dans sa nouvelle stratégie définie lors de son $25^{\mathrm{e}}$ congrès en février 1985; ligne que la déclaration de Roland Leroy résume : " Nous ne sommes plus dans la majorité depuis que nous ne sommes plus au gouvernement ${ }^{38}$." Le choix de la Bretagne, pour le dernier voyage avant les échéances déterminantes de mars 1986, n'est donc pas un hasard. François Mitterrand y vient pour renforcer ses amis politiques et pour tenter l'ouverture au centre.

36. Libération, le 9 octobre 1985.

37. Ouest-France, 5-6 octobre 1985.

38. BECKER, Jean-Jacques, Crises et alternances (1974-1995), Nouvelle histoire de la France contemporaine n 19, Paris, Le Seuil, coll. " Points-Histoire ", 1998, p. 330. 


\section{Au centre des préparatifs : le chef de Cabinet de l'Élysée et le Préfet}

Cette visite présidentielle s'inscrit pleinement dans le type même du grand voyage en Bretagne à vocation politique. Il vient donc après ceux de De Gaulle en 1960 et 1969 et celui de Valéry Giscard d'Estaing en 1977. Il s'agit pour le Président de ratisser une région durant deux jours avec la volonté d'aller au contact, de rencontrer les forces vives, de voir des équipements ou des entreprises symboliques. Cela entre dans une politique de communication politique et de contacts entre le Président et les Français. Ces déplacements sont très lourds à monter ainsi que le précise Jean Glavany qui fut en charge de les organiser durant le premier septennat de François Mitterrand :

"Ce voyage en Bretagne a demandé trois ou quatre mois de travail pour à la fois répartir harmonieusement le voyage entre les quatre départements bretons, entre les différents secteurs, aussi d'un point de vue politique. C'est le fruit d'échanges subtils pendant plusieurs mois : il s'agit d'étudier les dossiers de la région, de savoir sur quels sujets les gens attendent que le Président se prononce. C'était des voyages extrêmement lourds, extrêmement préparés et extrêmement travaillés ${ }^{39}$."

Lorsque l'Élysée a pris sa décision, le chef de Cabinet met en œuvre le projet. Il est l'homme orchestre : il se voit proposer des programmes par le Préfet, il filtre, et fait des choix qui doivent incarner la thématique générale du voyage. Erik Orsenna, qui rédigeait alors les discours de François Mitterrand, indique que :

"Pour les voyages en province, tout nous venait des Préfets. Et je travaillais en étroite collaboration avec Jean Glavany, le chef de Cabinet. C'est lui qui, avec les élus et Matignon, proposait les thématiques ${ }^{40}$."

Le chef de Cabinet, qui se voit proposer au départ par le Préfet une dizaine d'étapes, doit compresser ce programme en trois ou quatre visites. Il doit alors faire preuve de beaucoup de diplomatie pour expliquer à des élus socialistes la non venue présidentielle. Et il doit faire accepter les vues de l'Élysée aux maires qui souhaiteraient pouvoir se montrer dans une position valorisante, aux côtés du Président lors d'un bain de foule, ou mieux parcourant avec lui les rues de leurs cités. C'est un exercice très difficile car il doit prendre en compte les données politiques locales, les éléments de l'identité économique du département, les invitations des maires et des acteurs économiques. Jean Glavany dirige les réunions avec les services de l'État et se rend sur le terrain afin de vérifier la faisabilité du programme :

"On échafaude avec le Président un programme précis, puis on va sur place dans une mission préparatoire pour faire la répétition générale du

39. Entretien avec Jean Glavany, député des Hautes-Pyrénées, ministre de l'Agriculture et de la Pêche (1998-2002), et ancien chef de Cabinet du Président François Mitterrand de 1981 à 1988, le 17 janvier 2005.

40. Lettre à l'auteur, 18 décembre 2003. 
voyage pour aller mettre les pas dans les pas du Président, voir comment ça va se passer, voir les temps de transport, les risques, etc. À l'issue de cette mission préparatoire, on propose des corrections éventuelles ${ }^{41}$. "

La préfecture réalise alors un important travail de préparation. Une monographie présentant le département est rédigée : elle présente les aspects géographiques, historiques (les grands hommes du département), économiques, et les grandes villes du Finistère. Les dossiers d'actualité sont recensés. On y trouve aussi les notices biographiques des élus (maires des villes principales et parlementaires) et celle du Préfet. Il y a également une présentation de l'évolution politique et électorale depuis 1945. On y lit d'ailleurs, qu'il est " remarquable d'observer, dans le Finistère, combien sont souvent proches les modes de pensée des responsables locaux, centristes de droite et catholiques progressistes de gauche ${ }^{42}$ ». Ce commentaire vient confirmer que la venue présidentielle s'inscrit bien dans un territoire où le Président vient essayer de séduire les centristes.

À côté des problèmes liés à la sécurité du Président, il y a des risques plus classiques : les manifestations syndicales. Le corps social profite de cette visite pour agir et médiatiser des problèmes économiques et sociaux. Le rôle des Renseignements Généraux est donc de mesurer ce risque et de tenter de le limiter en annonçant à l'Élysée, via le Préfet, les dossiers conflictuels. Des demandes d'audiences des syndicats affluent dès que l'annonce de la visite est officielle; entretiens que « le Président refuse systématiquement car sinon il serait obligé de les recevoir tous ${ }^{43}$ ". Du coup, le Président se déplace avec une quinzaine de conseillers techniques dont la fonction est justement de recevoir ces délégations syndicales.

\section{L'annonce de l'événement}

La presse annonce le programme de la visite et tente de dégager la thématique du voyage. Pour Ouest-France, cette visite se fera, entre histoire (la résistance à Guimaëc) et pauses futuristes (Ifremer, Bolloré-technologies), et souligne les oubliés du jour : le monde paysan et la Marine nationale. Le Télégramme demande à ce que la population accueille avec courtoisie le Président Mitterrand sur cette terre d'opposition.

" [...] Nous vivons dans un pays de liberté où existent maints moyens légaux d'exprimer son opinion sans avoir recours aux violences verbales ou matérielles. Chez nous, nul n'est obligé d'aller applaudir quelqu'homme d'État que ce soit. Chacun peut même, en toute sérénité, ignorer la présence de cet hôte. Mais on se doit de l'accueillir avec correction. Car enfin, il n'a pas imposé sa visite, il a répondu à une invitation de ceux que le suffrage universel a désignés pour représenter la nation. [...] Ce n'est pas servir les

41. Entretien avec Jean Glavany, le 17 janvier 2005.

42. Arch. mun. de Quimper, 11 W 7, Présentation du Finistère, document préfectoral, p. 19.

43. Entretien avec Jean-Paul Palmarini, Directeur départemental des Renseignements Généraux dans le Finistère lors de la venue de Mitterrand en 1985, le 30 avril 2004. 
institutions de la République que de huer, injurier, molester ceux qui les incarnent $[\ldots]^{44}$."

On le voit bien ici, la visite présidentielle est un événement légitimant. Ainsi, cet article rappelle-t-il que le visiteur annoncé est le chef de l'État, légitime, donc respectable. On peut également y lire un appel aux mécontents : servir les institutions c'est aller voter pour l'opposition en mars prochain.

Les maires appellent aussi à la courtoisie républicaine. Celui, gaulliste, d'Ergué-Gabéric semble satisfait d'accueillir le Président socialiste :

"Maire républicain, j'accueillerai volontiers le chef de l'État. Je n'ai pas les mêmes options mais je suis légaliste et nous vivons dans le même pays. Je pense en effet que les différentes collectivités depuis l'État jusqu'à la commune doivent vivre en harmonie ${ }^{45}$."

Autre exemple de communion républicaine : le député socialiste Jo Gourmelon et le maire RPR de Brest, Georges Kerbrat, annoncent qu'ils plaideront les mêmes dossiers en faveur de la ville et de l'arsenal. À Morlaix, Jean-Jacques Cléach encourage les habitants à pavoiser et à montrer la meilleure image de leur ville. Mais face à l'annonce de la participation de plusieurs adjoints (PCF et PSU) à une manifestation hostile à Mitterrand, il prévient : " [...] En fonction de ce qui se passera, je serai amené à en tirer les conséquences. Il y a des moments où l'on doit s'unir ${ }^{46}$. "Si les maires mobilisent la population, le corps social s'organise aussi : la presse se fait le relais des appels à manifester de la CGT, du PSU, des syndicats enseignants; tandis que le ministre de l'Agriculture annonce qu'il recevra la FDSEA, le CDJA et l'UDSEA. Ainsi, mesure-t-on déjà, avant même l'arrivée du Président Mitterrand combien sa venue vient renforcer sa légitimité car c'est vers lui que l'on se tourne pour évoquer les problèmes.

Un classique de la communication présidentielle est l'interview à la presse régionale la veille du déplacement. En octobre 1985, Mitterrand répond à Pierre Le Marc dans les colonnes d'Ouest-France. Les sujets abordés touchent à la fonction présidentielle, à la défense, à la future campagne électorale. Mais le thème central est un éloge de la région qu'il vient visiter : « La Bretagne n'a pas à avoir de complexes. Elle est la région d'Europe qui a accompli les progrès les plus remarquables depuis trente ans. Et peutêtre celle qui, aujourd'hui, a les plus grandes potentialités ${ }^{47}$. " Pour Pierre Le Marc, ce voyage présidentiel est bien un " test pour une future cohabitation ». La posture prise par François Mitterrand est celle du " Président rassembleur".

44. Le Télégramme, 7 octobre 1985.

45. Ouest-France, 7 octobre 1985.

46. Ouest-France, 8 octobre 1985.

47. Ouest-France, 7 octobre 1985. 


\section{Une posture : le "Président rassembleur» sur deux lieux de mémoire}

Pour apparaître légitime dans le cadre d'une configuration inédite de cohabitation, le Président doit tout faire pour être reconnu comme le Président de tous les Français. Deux lieux de mémoire viennent appuyer cette posture : Beg-an-Fry et l'île de Sein.

Ce 8 octobre 1985, le Président de la République vient au rendez-vous de son histoire. Car c'est par Beg-an-Fry, à Guimaëc, que dans la nuit du 26 au 27 février 1944 Mitterrand alias " Monsieur Jacques ${ }^{48}$ " est rentré de Londres. Accueilli par les résistants locaux, il est pris en charge pour la nuit et amené le lendemain jusqu'à Morlaix où il prend un train pour Paris. Cet endroit est devenu un classique des visites mitterrandiennes dans le Finistère car il s'y est déjà rendu à deux reprises, en 1954, il est alors Ministre de l'Intérieur dans le gouvernement de Pierre Mendès France, puis en 1978 en tant que Premier secrétaire du Parti socialiste.

On peut découper cette étape en trois séquences. Il y a tout d'abord, la rencontre pleine d'émotion avec des gens exemplaires parce que " drôlement courageux ${ }^{49}$ ! ». Il s'agit des anciens du réseau "VAR ». Il rencontre : Aristide Sicot, responsable des plages, la veuve et la fille de son convoyeur Mercier, et les sœurs Jacob qui l'ont hébergé à l'époque. En souhaitant les revoir, il témoigne de sa fidélité envers ces personnes qui l'aidèrent dans ces années sombres. Aujourd'hui, c'est en pleine lumière qu'il salue leur courage. Ainsi, rend-t-il hommage, à travers elles, à l'action de la résistance bretonne. Le deuxième acte est constitué par le récit, par lui-même, de son retour en France. Il raconte aux journalistes cette nuit du 26 au 27 février 1944 :

"C'était par une nuit sans lune. Pour tout bagage, les Anglais m'avaient confié un revolver et une boule de cyanure. Je me souviens aussi du rocher derrière lequel s'était abritée la vedette anglaise. Il me semblait plus haut, sans doute à cause de la marée. Je me rappelle aussi le trajet en barque jusqu'à la plage. En pleine nuit, ça semble long. Et puis, ce sentier abrupt qu'il a fallu grimper pour rejoindre le refuge. Voilà, j'étais jeune ${ }^{50}$ !»

Le souvenir est précis et souligne les risques inhérents à ce type d'opération en temps de guerre. Mais, il est bien plus que cela : si la mémoire du Président est si fidèle, c'est sûrement parce que cette nuit fut un moment important pour lui. Et parce qu'ainsi, il mêle habilement son histoire personnelle, celle du capitaine Morland, à la grande Histoire, celle de la résistance française. Le troisième acte est la rencontre avec un paysage et un territoire. On connaît la passion de Mitterrand pour la géographie de la France; pas seulement électorale. Revenu sur ce site de Beg-an-Fry, il juge

48. PEAN, Pierre, Une Jeunesse française, François Mitterrand 1934-1947, Paris, Fayard, 1994, p. 380.

49. Le Monde, 9 octobre 1985.

50. Ouest-France, 9 octobre 1985. 
que " c'est vraiment un bel endroit mais je n'avais pas eu le temps de l'admirer à cette époque ${ }^{51} »$. Plus loin, il montre qu'il n'est pas dans un lieu anonyme et qu'il connaît ce territoire : François Mitterrand surprend tout le monde en interpellant le maire de Guimaëc : "Alors, si je me rappelle bien, devant, c'est Locquirec, et là, derrière, c'est Saint-Jean-du-Doigt, le pays de Tanguy-Prigent ${ }^{52}$ ?"

Cette étape du voyage présidentiel est donc un double retour aux sources. Il y a tout d'abord les retrouvailles avec des gens exemplaires qui témoignent de son passé de résistant ${ }^{53}$ mais il y a aussi l'hommage rendu à cette terre du Trégor, ancrée à gauche, qui fut la circonscription de Tanguy-Prigent. Cette séquence est aussi pour lui l'occasion de faire cette remarque à Raymond Marcellin; petite phrase reprise par toute la presse nationale, à l'exception du Figaro : "Tous les Présidents de la République ne sont pas passés par là ${ }^{54}$..."

Ce premier acte de la visite du département s'appuie sur son histoire personnelle et entend rassembler les Français autour de ces valeurs, transcendantes, acceptées par tous, qui apparaissent en filigrane et que l'on peut regrouper autour de mots-clés en forme de binômes : Résistance-courage; fidélité-émotion; histoire-territoire. Mais s'agit-il aussi de faire entrer Beg-an-Fry dans la " Mitterrandie ", cette terre qui se parcourt et où " tout concourt à l'élaboration de la même œuvre : la mise en place de matériaux pour un culte posthume ${ }^{55}$ "? Le site de Beg-an-Fry est-il, pour François Mitterrand, un lieu de mémoire complémentaire à la roche de Solutré; ce " haut lieu " mitterrandien, dont l'ascension, chaque lundi de Pentecôte, depuis 1946, en a fait un " lieu exemplaire " destiné à pérenniser le souvenir de son action ${ }^{56}$. Car force est de constater qu'à Solutré, comme à Guimaëc, la mémoire entretenue est la même : c'est celle des " années noires " et de la résistance. Et c'est un même hommage qui est rendu à la fidélité et à l'attachement à ces gens avec lesquels il s'était battu.

"Le Président s'est toujours montré attaché aux monuments et aux lieux qui portent en eux une part de la mémoire nationale ${ }^{57}$ " souligne Marc Abélès. Si à Guimaëc, Mitterrand a rappelé sa trajectoire personnelle, à Sein il vient sur les traces du Général de Gaulle. Le chef de la France Libre s'est rendu à deux reprises sur cette île dont il disait à Londres en juillet 1940 que «C'est le quart de la France. » Le 28 août 1946, il y était venu remettre

51. Le Télégramme, 9 octobre 1985.

52. Ouest-France, 12 octobre 1985.

53. Jean Glavany remarque que : " Mitterrand ne détestait pas rappeler sa résistance pour montrer que dans la polémique il y avait une grande part de mensonge et d'aveuglement " (entretien le 17 janvier 2005).

54. Libération, 9 octobre 1985.

55. FLEURDORGE, Denis, Les Rituels présidentiels, Paris, PUF, 2001, p. 216.

56. ABELES, Marc, « François Mitterrand et la roche de Solutré », dans CREPU, Michel (dir.), Hauts lieux, op. cit.

57. ABELES, Marc, "Symbolique présidentielle et culture républicaine " dans AGULHON, Maurice (dir.), Cultures et folklore républicains, op. cit., p. 216. 
en personne la croix de la Libération en hommage aux 133 hommes qui s'étaient engagés dans la France Libre dès juin 1940. Lors de son grand voyage politique de septembre 1960, de Gaulle inaugure le monument aux Français Libres. Pour Maurice Agulhon, c'est un geste fort :

"Le Général n'est pas allé inaugurer partout, loin s'en faut. On peut retenir comme significatif qu'il ait tenu à présider l'inauguration, à l'île de Sein, d'un monument typiquement "France libre" et consistant naturellement en une croix de Lorraine ${ }^{58}$."

Pour Ouest-France (8 septembre 1960) cette visite fut " une fête bretonne, une fête du souvenir et une fête de famille " lors de laquelle " patriotisme et foi catholique " furent associés. Il est clair que pour tous, là, " le Président de la République retrouve les souvenirs du Général de Gaulle ${ }^{59}$ ". Les retrouvailles des Sénans et de De Gaulle furent chargées d'émotion : tous les hommes portaient l'insigne de la France Libre au revers du veston, et les femmes portaient la Jibilinen, cette coiffe à deux pans des femmes en deuil. Cet hommage du chef de la France Libre vient nourrir le mythe gaulliste de cette île, terre de courage et exemple pour la France entière. Les successeurs de De Gaulle à l'Élysée ne se sont pas rendus sur l'île en visite officielle. François Mitterrand est donc, en octobre 1985, le premier Président à le faire depuis 25 ans.

Le programme de la visite présidentielle est simple : dépôt d'une gerbe au pied du mémorial aux Français libres, bref passage en mairie pour signer le livre d'or, déjeuner à l'auberge des Sénans puis départ pour Lorient. Si, sur l'île, le déplacement est calme car il n'y a pas de manifestants de la CGT et du PCF, le Président socialiste est en terre gaulliste. Certains habitants font passer le message aux journalistes qui rapportent ces remarques : "Ici on n'est ni de gauche ni de droite, on est gaulliste forcément " ou " le peuple de gauche n'est pas ici ${ }^{60}$ ". Il faut dire qu'en mai 1981, Valéry Giscard d'Estaing y avait obtenu $81 \%$ des voix. À la mairie, le portrait officiel du Président qui tient la place d'honneur est celui du fondateur de la $V^{e}$ République; celui de Mitterrand étant accroché dans un coin de la salle des délibérations. Après avoir rendu hommage aux Sénans de 1940, le Président tient à préciser que " [leur] histoire n'a pas commencé le 18 juin 40 ». Et il souhaite inscrire sa visite dans le temps long : "Pour nous gaullistes, cette visite à Sein ne fut pas une provocation mais plutôt un geste de respect. Mitterrand avait le sens de l'histoire, et il savait qu'au-delà du 18 juin 1940 , cette île a une histoire et que cette histoire continue ${ }^{61}$."

La visite de l'anti-gaulliste sur cette terre mythique est donc perçue comme un hommage. D'ailleurs, la presse et les personnalités qui accompagnèrent le Président soulignent que l'accueil fut simple mais cordial. L'article

58. Maurice AgulHon, De Gaulle, Histoire, symbole, mythe, Paris, Plon, 2000, p. 90.

59. Le Monde, 9 septembre 1960.

60. Ouest-France, 9 octobre 1985.

61. Entretien avec Charles Miossec, Président du Conseil Général du Finistère (19881998) et député RPR (1978-2002), le 9 mai 2003. 
d'Ouest-France du 9 octobre relatant la visite présidentielle sur l'île est titré simplement : "Sein, une page tournée ». S'agit-il de l'événement lui-même ou de l'opposition frontale dans laquelle se trouvait François Mitterrand vis-àvis du Général de Gaulle? Car le Président déclare à la mairie :

"Ce n'est pas sans émotion que je viens ici, cette île porte une telle charge d'Histoire. J'ai rencontré ici des Sénans, ceux de 40 et leurs enfants. Ici, les hommes sont habitués à exercer un dur travail. Leurs parents leur ont appris à lutter pour vivre ${ }^{62}$."

François Mitterrand sculpte son image de rassembleur en venant sur ce lieu de mémoire gaulliste rendre hommage aux Français Libres. En apportant, en quelque sorte, sa reconnaissance à cette famille politique, il épouse pleinement la thématique consensuelle du voyage.

\section{Une visite pour montrer ce que pourrait être la cohabitation?}

Ce déplacement permet de montrer à tous que la cohabitation qui s'annonce après mars 1986 est déjà le quotidien du Président. Car dans le Finistère, Mitterrand est reçu à Brest, Ergué-Gabéric, Sein et Quimper par des maires qui sont tous RPR. Ainsi, le Président dresse-t-il un mode d'emploi de la cohabitation. Il rappelle qu'il ne doit pas y avoir de confusion sur le rôle et les compétences du Président. Il se réfère à la constitution, " qu'il connaît d'autant mieux qu'il ne l'a pas votée alors que d'autres semblent pressés de la bafouer, en pointant le doigt sur Raymond Barre sans le citer $^{63}$ ". En effet, l'ancien Premier ministre a déclaré dès avril 1984 que cohabiter serait contraire à l'esprit des institutions. Ainsi, Mitterrand se présente déjà comme un intermédiaire entre les maires de droite, qui l'accueillent, et le gouvernement. Et lorsque le maire de Quimper lui demande de pousser quelques dossiers concernant sa ville, il tient à prendre une hauteur toute présidentielle :

" Je ne ferai pas le tour, Monsieur le Maire, des questions que vous avez traitées. D'abord elles ne sont pas de mon ressort, mais bien entendu, il importe que j'en sois informé et vous avez eu raison de m'en saisir. [...] Si je m'en tenais au terme exact de la constitution, $[\ldots]$ je dirais que ce qui est vrai c'est que c'est au gouvernement de gouverner ${ }^{64}$."

À côté de la délimitation des pouvoirs du Président, Mitterrand définit aussi le terrain consensuel sur lequel chacun peut se retrouver : lutte contre le chômage, modernisation du pays et défense nationale. Ces trois piliers semblent donc être une base sur laquelle il souhaite pouvoir rassembler les Français : "Lorsqu'il s'agit de grandes tâches, soyons capables de les faire ensemble $^{65}$. " Cette tentative de définition du mode d'emploi de la future

62. Ouest-France, 9 octobre 1985.

63. Libération, 9 octobre 1985.

64. Arch. mun. de Quimper, $11 \mathrm{~W}$ 7, allocution du Président Mitterrand à Quimper, le 8 octobre 1985 .

65. Le Figaro, 9 octobre 1985. 
cohabitation n'est pas innocent lorsqu'il se fait devant les élus de l'opposition et en particulier devant des maires et des parlementaires gaullistes.

\section{L'éloge de la politique menée par Marc Bécam}

La visite de Quimper est le moment fort de cette stratégie. François Mitterrand fait l'éloge de la politique culturelle menée par la municipalité de Marc Bécam. Cela fait les gros titres de la presse régionale : " Coup de chapeau et coup de cœur pour Quimper " (Le Télégramme, 9 octobre 1985); "Le coup de pouce à la politique culturelle de Marc Bécam " (Ouest-France, 9 octobre 1985). Marc Bécam, ancien secrétaire d'État aux Collectivités locales du Président Giscard d'Estaing, intéresse l'Élysée depuis déjà quelques mois. En juillet 1984, le nouveau Premier ministre, lui avait proposé de devenir son conseiller à la Sécurité mais la discipline de parti l'obligea alors à décliner cette proposition. Dès qu'il apprend le projet de voyage du Président de la République, le maire de Quimper fait savoir qu'il serait ravi de l'accueillir dans ses murs. Cette visite peut donc servir le Président mais aussi Marc Bécam qui peut ainsi se placer dans l'éventualité d'une cohabitation : " On imagine très bien ce RPR à la fibre chabaniste siéger dans quelque ministère ${ }^{66}$. " Et l'accueil du sénateur-maire va bien au-delà de la tradition de courtoisie républicaine :

"Monsieur le Président de la République, vous êtes le bienvenu à Quimper.

C'est un grand honneur pour moi de vous le dire, au nom des Quimpérois, de toutes les personnes rassemblées ici et tout particulièrement les membres du Conseil municipal.

Le dialogue qui est l'honneur de la République, est ici une qualité qui franchit bien des barrières, même s'il est naturel que chacun garde ses convictions ${ }^{67}$."

De son côté, le Président de la République dresse un portrait flatteur de cette ville dirigée par une municipalité d'opposition :

"Sur le plan de la culture, vous avez réellement bien travaillé. Et j'ai voulu dire au passage ce que l'État avait fait parce que c'est mon devoir de le dire, mais, bien entendu, ce n'est pas l'État qui va dire tout à coup à une ville : "Vous allez donner un élan à la culture locale au point de devenir un centre d'intérêt national!" C'est parce que l'initiative est venue de là et que sans doute vous-même, Monsieur le Maire, vous avez trouvé autour de vous et dans cette ville des gens qui s'en sentaient la capacité $[\ldots]^{68}$."

La posture de " Président rassembleur " est d'autant plus manifeste à Quimper qu'elle reçoit en écho l'accueil zélé de ce maire atypique que chacun présente comme un archétype de la race des cohabitants. Plus géné-

66. Ouest-France, 5-6 octobre 1985.

67. Arch. mun. de Quimper, 11 W 7, allocution du maire de Quimper, 8 octobre 1985.

68. Arch. mun. de Quimper, 11 W 7, allocution du Président Mitterrand à Quimper, 8 octobre 1985 . 
ralement, l'accueil républicain des maires de droite, qui n'attaquent pas la fonction présidentielle, vient renforcer la légitimité du Président. Il contraste avec l'opposition de la rue, organisée par la CGT et le PCF; agitation qui dresse le constat de divorce entre une gauche " raisonnable " et l'autre. Du coup, ce dialogue républicain quimpérois vient accentuer la virulence de la CGT vis-à-vis du Président socialiste : "Mitterrand-Bécam : même combat $^{69}$ ", clament les militants CGT après avoir crié " Mitterrand, trahison. "

\section{Se déplacer pour le Président, c'est aussi l'occasion de renouer avec les siens}

Si François Mitterrand entend préparer la future cohabitation en essayant de rassembler les Français autour de sa personne et de sa fonction, ce déplacement dans le Finistère est également l'occasion pour lui de prôner l'union des socialistes à quelques jours de leur congrès de Toulouse. Sa posture de "Président rassembleur " lui interdit de se montrer en chef de parti, en chef des socialistes. Mais il précise toutefois qu'il se sent " non pas le Père, mais l'ancien responsable de ce grand mouvement qui durera longtemps après moi. Je désire les voir unis ${ }^{70}$ ".

Avec 30 \% des mandats pour le congrès de Toulouse, Michel Rocard a fait une percée dans le parti. Dans le Finistère, où les rocardiens sont très bien implantés, ils en rassemblent $63 \%$. C'est dans ce contexte de politique interne au PS que Mitterrand saisit l'occasion de se rapprocher du plus éminent des socialistes finistériens, rocardien de surcroît : Louis Le Pensec. Il s'agit de renouer après la rupture de mars 1983 où n'acceptant pas de voir son ministère rabaissé au rang de secrétariat d'État, rattaché au ministère des Transports, Louis Le Pensec choisit de quitter l'équipe gouvernementale et " le ministre tire sa révérence ${ }^{71}$ ». Depuis, il pensait qu’il " était exclu que François Mitterrand [l']appelât à nouveau un jour au gouvernement ${ }^{72}$ ". Et durant les deux années qui suivirent sa démission, il n'eut aucun contact avec le chef de l'État. Puis, lors de la préparation de ce voyage en Bretagne, l'Élysée lui fit savoir que Mitterrand demandait à le voir : " Je comprends alors que ce déplacement présidentiel va être aussi l'occasion de recoller les morceaux entre le Président et son ancien ministre de la Mer ${ }^{73}$. "Le signe présidentiel est envoyé à Lorient, Mitterrand lui rend un hommage appuyé pour son action au sein du gouvernement entre 1981 et 1983. Il s'entretient alors avec lui : " Vous êtes un passionné, hein! [...] J'ai beaucoup regretté votre départ ${ }^{74}$. " Ainsi, le "Président rassembleur " n'oublie pas de renouer avec ceux de son camp; d'autant plus qu'il invite publiquement à l'union des courants. Il est entendu car le 13 octobre 1985, le parti refait son unité

69. Ouest-France, 9 octobre 1985.

70. Le Monde, 9 octobre 1985.

71. Le PENSEC, Louis, Ministre à bâbord, Rennes, Ouest-France, 1997, p. 192.

72. Ibidem, p. 199.

73. Entretien avec Louis Le Pensec, le 23 octobre 2003.

74. Le Pensec, Louis, op. cit., p. 199. 
lors de ce congrès qui apparaît être celui de la rénovation. Ce retour en grâce de Louis Le Pensec fut concrétisé en 1988 par sa nomination dans le gouvernement dirigé par son ami Michel Rocard : il redevient ministre de la Mer (mai-juin 1988) avant d'être nommé ministre des Dom-Tom (19881993) et de s'atteler au retour à la paix en Nouvelle-Calédonie.

\section{Un message :}

\section{la Bretagne est un exemple de la "France qui gagne »}

Durant les deux jours de ce voyage en Bretagne, François Mitterrand martèle le même message : il vient saluer les efforts d'adaptation, la modernisation de la Bretagne et les scientifiques qui innovent. Pour le faire passer, il fait un plaidoyer de son action depuis 1981 et vante les mérites de cette Bretagne qui gagne en s'appuyant sur deux entreprises exemplaires : Ifremer et Bolloré.

Le Président de la République désigne les responsables de ce qui a été manqué avant 1981, Raymond Barre, Jacques Chirac et Valéry Giscard d'Estaing, " ces experts, ces grands sages qui se sont trompés [...] et qui prétendent incarner l'avenir ${ }^{75}$ ". Lui, il est sûr que l'histoire rendra justice à son action depuis mai 1981 : "Ceux qui ont mis la main à la pâte en 1981 se trouveront plus vite qu'ils ne le croient validés dans leur action ${ }^{76}$. " Se faisant le chantre de la modernisation du pays, il lance un appel à l'effort général mais récuse le libéralisme au centre du programme économique de la droite pour l'après-1986 : " Ne croyons pas au laisser-aller qui ferait que la seule règle de la vie serait d'être plus fort que les autres et donc de les écraser au passage ${ }^{77}$. " La modernisation du pays doit être accompagnée d'ambitieuses réformes sociales; ce qui fait dire à Jean-Yves Lhomeau, du Monde le 9 octobre 1985, "Ainsi va la morale de l'histoire : M. Mitterrand est toujours socialiste..."

Si avec ce plaidoyer pour l'action menée depuis 1981, François Mitterrand défend son bilan, il entend également maintenir sa posture de "Président rassembleur ". L'économie est justement un thème qui lui permet de lancer un appel qui dépasse son camp. Et ces quelques phrases présidentielles sont déjà teintées de cohabitation : "Sachons dépasser ce qui risque de freiner l'action. Personnellement je me sens à l'aise avec chacun d'entre vous. Sur des questions comme l'emploi, la nécessité de modernisation de notre industrie, sur la formation et sur la défense, il existe en France un consentement sur lequel il doit être possible de se rassembler $^{78}$. " Prononcé à Brest, devant un maire RPR, ces paroles présidentielles sont un appel au " consentement ", au rassemblement, sur des sujets qui transcendent les clivages politiques.

75. Le Monde, 9 octobre 1985.

76. Ibidem.

77. Ibid.

78. Le Télégramme, 9 octobre 1985. 
Cette Bretagne est un bel exemple à donner à la France. Il loue son dynamisme et se présente en rassembleur des énergies; posture qui le fait apparaître au-dessus de la mêlée politicienne : "Un pays n'obtient rien sans courage, sans effort et sans volonté, autrement tout s'en va et tout lâche. Or, je suis là pour que la France tienne et qu'elle gagne ${ }^{79}$. "Cette thématique trouve sa concrétisation dans deux visites d'entreprises. Il s'agit ici d'une pédagogie présidentielle par l'exemple qui s'inscrit dans la tradition. Car déjà sous la III ${ }^{e}$ République, le chef de l'État en déplacement rendait visite à des manufactures célèbres pour leur production ou pour l'exemplarité de la gestion sociale de leurs employés. Il rend ainsi hommage au personnel et aux chercheurs de l'Ifremer, " à la pointe de Bretagne, c'est la Silicon Valley de la mer, manière Cinquième République ${ }^{80}$ ", c'est l'agence française pour l'océan. La visite de Bolloré-Technologies, à Ergué-Gabéric, s'inscrit aussi dans cette pédagogie par l'exemple; d'autant plus qu'elle permet de jouer sur deux axes complémentaires aux yeux du Président socialiste : la réussite industrielle et le modèle social. Le cortège visite le site et Mitterrand va à la rencontre des ouvriers. C'est l'occasion de souligner le bon climat social qui règne dans l'entreprise. D'ailleurs, si le député Poignant a joué les intermédiaires pour décrocher la visite présidentielle, c'est à la demande des délégués syndicaux de la CGT et de FO. Ceux-ci refusèrent de s'associer à la campagne nationale d'opposition frontale au patronat et au PS. Ils souhaitaient au contraire que la venue du chef de l'État permette à l'entreprise d'obtenir des aides nationales pour aider Vincent Bolloré. À travers cette visite, Mitterrand montre à la France un exemple de la Bretagne qui gagne et il met en lumière un type de patronat qui sait associer modernisation et progrès social.

\section{À la rencontre des Finistériens}

Un des buts des voyages présidentiels est de permettre la rencontre entre le Premier des Français et les citoyens. Ce déplacement d'octobre 1985 ne déroge pas à cette règle. Le Président rencontre tout d'abord les officiants puis se donne à voir aux spectateurs.

La mairie est par excellence le lieu républicain de la rencontre. Elle est décorée de "marqueurs " qui solennisent la venue présidentielle : tapis rouge à l'entrée, drapeaux tricolores dans les rues de la ville et sur la mairie. L'accueil est ancré dans l'identité bretonne avec des binious et la remise d'un bouquet de fleurs par des jeunes filles en costume traditionnel. Dès l'arrivée du Président, le maire devient l'homme clé : il l'accueille à sa descente de voiture et le fait entrer dans l'hôtel de ville. Il se fait alors maître des cérémonies et présente son conseil municipal avant de lire une adresse au chef de l'État et de lui remettre un cadeau. Le Président répond à l'intervention du maire puis signe le livre d'or.

79. Ouest-France, 9 octobre 1985.

80. Ouest-France, 5-6 octobre 1985. 
Le rituel d'accueil du chef de l'État est parfaitement respecté lors de ce voyage par les maires de l'opposition. Pas un élu ne souhaite faire d'incident devant le Président socialiste. Ce respect du protocole a un effet légitimant pour le chef de l'État. Car c'est lui qui est au centre des honneurs et c'est à lui que ces élus RPR adressent leurs demandes; montrant ainsi à tous qu'il détient la plus haute charge de la République. Les maires adressent au Préfet quelques jours avant la visite leur projet de discours. Il s'agit ainsi de préparer la réponse du Président mais aussi de contrôler l'usage de la parole devant le chef de l'État. Les maires interviennent souvent en deux temps : après avoir dressé un portrait de leur ville, ils évoquent les projets pour la cité dans lesquels l'État est partie prenante.

\section{La parole présidentielle}

À Quimper, comme à Brest, le Président ne fait pas d'effet d'annonce. Il enregistre les demandes et assure qu'il les transmettra au gouvernement. Mais, ce 8 octobre 1985, Mitterrand entend se servir de ses réponses pour développer la thématique générale du voyage : la Bretagne qui gagne; l'appel au consentement et au rassemblement sur la modernisation du pays; et la définition du rôle du Président dans l'hypothèse cohabitationniste. Le discours présidentiel peut être général sans ancrage précis avec la réalité de la ville où il est prononcé. Par exemple, à Morlaix, il rend hommage à la Bretagne et aux Bretons en faisant un appel à l'effort et à l'initiative locale. Ce type d'intervention est parfois créateur de frustration : "Ceux qui attendaient une nouvelle touchant à l'avenir de Morlaix en auront été pour leurs frais. Rien de cela dans le discours du Président de la République. [...] Il n'y eut aucune réponse précise aux souhaits émis par le $D^{\mathrm{r}}$ Cléac' $\mathrm{h}^{81}$. "

À Brest, il se sert du lieu pour faire une intervention générale sur la défense nationale dans un contexte de politique intérieure marqué par l'affaire du Rainbow Warrior coulé par la DGSE, les services secrets français, le 10 juillet 1985. Même si le nom de Greenpeace n'est pas prononcé, chacun comprend que Mitterrand cherche à se justifier sur cette affaire lorsqu'il réaffirme, dans la cité du Ponant, l'importance de la dissuasion nucléaire :

" Je n'admettrai pas que la France soit privée de quelque moyen que ce soit alors que quatre autres pays en disposent. La France doit montrer son intransigeance non pas en exigence ou insolence à l'égard de quiconque mais elle doit interdire aux autres d'empiéter sur la juste et normale souveraineté d'un pays... Quoi qu'on en dise, quoi qu'on fasse, nous continuerons notre route ${ }^{82}$."

La parole présidentielle peut être aussi une riposte aux attaques qu'il subit lors de ce déplacement en Bretagne. L'agitation qui l'accompagne dans chacune des villes visitées l'irrite et il profite de ces discours face aux

81. Le Télégramme, 9 octobre 1985.

82. Le Figaro, 9 octobre 1985. 
élus pour répondre à la CGT et au PCF qui l'accusent de trahison. À Quimper, la riposte est cinglante :

"Et lorsque j'entends les protestations, je m'interroge à ce sujet et j'ai envie de dire d'abord à ceux qui ne connaissent que cette façon de s'exprimer, que moi le commando, je connais, mais à l'époque c'était plus difficile, du moins c'était plus dangereux. [...] Je sais parfaitement d'où ça vient, pourquoi c'est fait, c'est-à-dire pour pas grand-chose. [...] Ça m'ennuie même d'entendre des braves gens s'égosiller pour rien du tout car il ne suffit pas de débiter un certain nombre de sornettes pour conduire la France à trouver la voie du progrès. Et moi je ne veux pas qu'elle s'enracine dans les destructions du passé qui ne feront qu'accroître le malheur des travailleurs. [...] Il y a ceux qui servent les travailleurs parce qu'ils préparent le lendemain qui commence aujourd'hui parce qu'il faut former les enfants aux disciplines et aux métiers qu'ils feront et non pas aux métiers qui n'existeront plus ${ }^{83}$."

Face à l'esprit commando qui anime l'opposition très forte de cette gauche en rupture avec les socialistes, Mitterrand évoque son passé de résistant afin de montrer que cela ne l'impressionne pas. Et il dresse le portrait de deux gauches : l'une, celle qu'il incarne, qui prépare l'avenir, et l'autre, celle qui manifeste et qui crie, qui refuse la nécessaire modernisation. Ce passage du discours quimpérois est salué par les élus socialistes et par ceux de la droite : les applaudissements ici sont symboliques. Car en réalité, cette agitation de la rue ne sert-elle pas la posture générale de ce voyage? Elle permet à Mitterrand de désigner les mauvais joueurs que sont Krasucki et Marchais et de montrer à l'opposition que sur certains sujets, déjà évoqués, un consentement, à défaut d'un consensus, est possible avec elle. La place choisie par chacun est symbolique : l'opposition reçoit le Président avec une courtoisie toute républicaine tandis que les élus communistes et ceux du PSU préfèrent la rue à la salle de réception.

\section{À la rencontre des gens}

Le Président se ménage toujours des moments pour aller au-devant des gens venus le saluer. Ces bains de foule sont fréquents lors de cette visite de 1985. Les photographies sont nombreuses, dans la presse régionale, qui le montrent serrant des mains face à une foule maintenue à distance par des barrières et par des policiers en grande tenue. La presse aime rapporter le témoignage des heureux qui ont touché le Président, comme cet ouvrier de Bolloré-Technologies : " Il m'a serré la main. Il m'a demandé ce que je faisais. Il a dit que nous avions de la chance de travailler ici ${ }^{84}$. " Instants fugaces d'une rencontre improbable entre un citoyen de base et le Président de la République avec qui il y a contact physique et paroles échangées. Cette anecdote, associée à la pratique du bain de foule, pose le problème de l'expression du lien citoyen dans le cadre de ces déplacements. Sous la III ${ }^{\mathrm{e}}$ République ce type de question irrite et embarrasse les

83. Arch. mun. Quimper, $11 \mathrm{~W}$ 7, allocution du Président à Quimper, le 8 octobre 1985. 84. Le Télégramme, 9 octobre 1985. 
républicains : «N'y a-t-il pas une forme de superstition païenne digne des régimes passés dans le fait de vouloir toucher la main d'un homme fût-il (ou à plus forte raison encore) le magistrat suprême de la République ${ }^{85}$ ?" Avec la Ve République et la personnalisation du pouvoir de ce régime, cette pratique ne semble plus soulever de problèmes. Au contraire, lorsque le Président ne va pas vers la foule il est immédiatement soupçonné de mépriser les citoyens venus à sa rencontre. Il y a bien un désir de Père en politique. Les Français veulent voir et toucher le pouvoir. Et ils lui demandent d'assumer " le rôle de l'État et en même temps les citoyens veulent sentir l'ombre du parasol, la protection du Président. Cela m'étonne terriblement de la part d'un peuple qui a coupé la tête de son roi ${ }^{86}$ " souligne Kofi Yamgnane.

\section{Les rituels d'opposition}

Face aux rituels consensuels avec les officiants, cette visite d'octobre 1985 est l'occasion de souligner l'importance des rituels d'opposition qui sont au rendez-vous de chaque étape du programme présidentiel. Les organisations syndicales mobilisent leurs adhérents afin de manifester leur rejet de la politique menée par les socialistes. Il s'agit ici pour elles de combiner symbolique et parole. Le rituel d'affrontement met en lumière les symboles de l'antagonisme : un peuple dans la rue, armé de banderoles, et criant des mots d'ordre qui interpellent le pouvoir. C'est une démonstration de puissance qui doit avoir une résonance médiatique démultipliée par la présence de journalistes de la presse nationale.

Pour que leur présence ne puisse pas passer inaperçue la CGT et le PCF utilisent des pétards, des feux de Bengale, des lancers de ballons, des lancers de tracts multicolores, des fumigènes, et leurs banderoles défient le Président avec ces inscriptions "Mitterrand trahison ", "Des emplois, pas des bombes ", "Chômage, ras-le-bol ". Comme le souligne Louis Le Pensec : "Face à ce type d'adversité et d'opposition, le Président ne doit pas défaillir et se doit d'aller au-devant des manifestants quand cela reste bon enfant ${ }^{87}$." Ces rituels d'opposition ne sont pas nouveaux et avec la Ve République, ils se font systématiques. La dernière visite du Général de Gaulle en février 1969 est ponctuée de manifestations; le contexte est alors toujours marqué par les suites de mai 1968. Pompidou puis Valéry Giscard d'Estaing doivent également faire face à des revendications sociales et à des manifestations importantes qui rassemblent 10000 personnes à Brest en novembre 1974 et autant à Quimper en février 1977.

85. MARIot, Nicolas, "Propagande par la vue. Souveraineté régalienne et gestion du nombre dans les voyages en province de Carnot (1888-1894) " Genèses. Sciences sociales et histoire, $\mathrm{n}^{\circ} 20$, septembre 1995, p. 24.

86. Entretien avec Kofi Yamgnane, le 10 juillet 2003.

87. Entretien avec Louis Le Pensec, le 23 octobre 2003. 


\section{Le protocole et le rituel}

En analysant le découpage de cette venue présidentielle on remarque que ce voyage de deux jours en Bretagne est marqué par une suite de séquences, souvent identiques, qui caractérisent, selon Claude Lévi-Strauss, le rituel : le morcellement et la répétition. Ainsi, assiste t-on, comme a pu le faire Marc Abélès à Nevers, à " la célébration d'un vaste rituel où la combinaison des paroles proférées, des gestes accomplis, des objets manipulés met en jeu la symbolique des relations entre le pouvoir politique et la société civile $^{88}$ ». Tout au long du déplacement et à chaque séquence, le point focal reste le Président vers qui tout converge et qui est le centre de toute action. Le protocole républicain met en valeur le chef de l'État. Et le Président use de ses ressources pour diviser ses opposants, rassurer les siens et tenter de rassembler les Français. Et si Norbert Elias a bien montré que sous l'Ancien régime "l'étiquette est un instrument de domination ", Yves Déloye souligne que le protocole, qui classe, hiérarchise et dévoile la suprématie des uns et la domination des autres, est bien aujourd'hui encore une " grammaire de l'assentiment politique ${ }^{89}$ ». François Mitterrand nous en donne une démonstration à travers cet exemple de voyage de souveraineté.

\section{La résonance de l'événement}

La résonance de l'événement dans la presse est un enjeu majeur. Les journalistes politiques des grands quotidiens nationaux qui sont accrédités auprès de l'Élysée suivent le Président durant tout le voyage. Un ou deux journalistes de la presse régionale rejoignent cette équipe. LouisRoger Dautriat, qui en fit partie, témoigne.

"Lors de cette visite d'octobre 1985, le travail de la presse fut grandement facilité. Il y avait deux points de presse par jour, le Président était assez accessible, on sentait que la communication présidentielle était au centre de ce voyage, que c'était là l'essentiel pour l'Élysée ${ }^{90}$."

Pour ce voyage, il est possible de dégager, à partir des titres des articles, cinq grandes thématiques reprises par les grands journaux parisiens :

1) Mitterrand défend la modernisation du pays

"Le chantre de la modernisation ", Le Monde, 9 octobre 1985

"La modernisation doit être accompagnée d'ambitieuses réformes sociales ", Le Monde, 10 octobre 1985

2) L'agitation menée par le PCF et la CGT

"Le défi des commandos de la CGT », Le Matin, 9 octobre 1985

88. ABELES, Marc, "Inauguration en gare de Nevers. Pèlerinage à Solutré ", Les temps modernes, 1987, p. 81.

89. Deloye, Yves, " Le protocole ou l'ombre du pouvoir politique " dans Deloye, Yves, HAROCHE, Claudine, IHL, Olivier, Le protocole ou la mise en forme de l'ordre politique, Paris, L'Harmattan, 1996, p. 63.

90. Entretien avec Louis-Roger Dautriat, journaliste politique au Télégramme, le 2 avril 2004. 
" Face à face bruyant Mitterrand-CGT ", Le Figaro, 9 octobre 1985

"Moi, les commandos, je connais ", Le Monde, 10 octobre 1985

" Coup de colère de Mitterrand contre la CGT ", Le quotidien de Paris,

9 octobre 1985

3) Le soutien affiché au Premier ministre

" Blanc-seing présidentiel pour Laurent Fabius ", Le Matin, 9 octobre 1985

" Mitterrand donne quitus à Fabius ", Libération, 9 octobre 1985

"Mitterrand manifeste sa confiance à Fabius ", Le Parisien Libéré,

9 octobre 1985

4) Les pouvoirs du chef de l'État et la future cohabitation

"Les pouvoirs du Président ", Le Monde, 10 octobre 1985

"Plus cohabitationniste que moi, tu meurs... ", Le Figaro, 11 octobre 1985

5) Le passé de résistant

"Dans les pas du résistant Morland ", Le Monde, 9 octobre 1985

Ces titres s'intègrent à la thématique générale du voyage qui jouait sur la capacité de François Mitterrand à rassembler les Français. Il profite de ce déplacement pour adresser plusieurs messages ciblés. Il distingue la gauche raisonnable de celle qui " débite des sornettes ". La présence assez complice, à ses côtés, de Raymond Marcellin, en tant que Président du Conseil Régional de Bretagne, "irrite certains socialistes, car pour eux il reste l'adversaire politique. Mais c'est une manière de dire aux jeunes socialistes qu'il faut apprendre à composer, que le pouvoir change ${ }^{91}$ ". À droite, il envoie le message cohabitationniste. Et l'utilisation du protocole lui permet de valoriser sa recherche de légitimité mais aussi de montrer son autorité et sa puissance face à l'agitation de la rue. À l'ensemble des Français, il tente d'adresser un message rassurant de rassemblement en valorisant la réussite exemplaire de certains secteurs économiques.

\section{Une conséquence du voyage présidentiel : des crises municipales}

Ce déplacement n'est pas sans effets au sein des villes dirigées par des maires PS. Car partout ce fut une gauche désunie qui a accueilli Mitterrand. Et à Rennes, Saint-Brieuc, Morlaix et Lorient, les cicatrices sont vives. Pour Jean-Jacques Cléach, " il n'y plus de majorité municipale ${ }^{92}$ " à Morlaix. Le fait que ce rituel consensuel n'ait pas été respecté est pour lui inacceptable : «Si le Président avait été de droite, je l'aurais reçu comme les municipalités de l'opposition, à Brest et à Quimper, ont reçu Mitterrand. Le PC et le PSU n'acceptent pas la République ". Puis, il ajoute qu'il recevait "Mitterrand au nom de tous les Morlaisiens. J'estime donc que la manifestation était autant dirigée contre le chef de l'État que contre moi ${ }^{93}$ ". Du côté des adjoints PC et PSU, auxquels Jean-Jacques Cléach a retiré les délé-

91. Entretien avec Louis Le Pensec, le 23 octobre 2003.

92. Le Télégramme, 10 octobre 1985.

93. Ibidem. 
gations, il s'agit d'expliquer leur position en ménageant le maire : « Les élus communistes se sont trouvés aux côtés des travailleurs qui manifestent... En aucun cas, il ne s'agissait pour eux de mettre en cause la politique municipale et le maire ${ }^{94}$. "Cette affaire laisse des traces. Et pour Jean-Jacques Cléach : "Certains ont estimé que c'est à cause de cela que nous avions perdu les municipales de mars $1989^{95}$."

Ce déplacement en Bretagne, à quelques mois des élections, entrait bien dans le cadre de la préparation des législatives. La posture du «Président rassembleur " allant sur des lieux de mémoire consensuels, se présentant en défenseur de la France qui gagne devait renforcer son image de Président en charge de l'essentiel. Mais il s'agissait d'un exercice difficile. Car François Mitterrand devait réaffirmer son identité socialiste et avoir un discours mobilisateur pour la gauche tout en lançant un appel à l'unité nationale autour de sa personne. Au terme de ces deux jours en Bretagne, le Président pouvait espérer sortir de ce voyage avec un surcroît de légitimité et peut être aussi avec quelques suffrages de centristes bretons.

Ces deux exemples de visites présidentielles témoignent des ressources que procure au Président le déplacement en province. Pour Mitterrand et l'équipe de l'Élysée, le voyage présidentiel doit produire de la légitimation et conforter son pouvoir ${ }^{96}$. Sa position est renforcée grâce aux rituels qui le montrent comme le premier des Français et par le protocole qui valorise les amis politiques tandis qu'il domestique les opposants. Car, que se soit en chef des Armées ou en " Président rassembleur ", le déplacement a toujours une motivation politique. En 1981, il lui permet de rassurer sur la capacité de la gauche à gouverner et à s'inscrire dans la durée tandis qu'en 1985 il sert à se préparer à l'après mars 1986 en cherchant à atténuer une défaite " qui semblait écrite ${ }^{97}$ ". Le déplacement reste aussi un outil utilisé par l'Élysée afin de maintenir le lien avec les Français et pour développer une stratégie de communication présidentielle en s'appuyant sur des lieux, des gens, et des entreprises modèles. Il est frappant de constater aussi que cette posture de "Président rassembleur " annonce déjà le thème de " la France unie » qui fut au cœur de la campagne présidentielle de 1988.

\section{1-1985 : deux usages de la visite présidentielle en province}

Analyser ces deux visites de François Mitterrand dans le Finistère, c'est donc s'interroger sur le sens de ces déplacements mais c'est aussi questionner " nos conceptions mêmes du pouvoir et de la souveraineté, des rapports entre gouvernants et gouvernés ${ }^{98}$ ".

94. Ouest-France, 15 octobre 1985.

95. Entretien avec Jean-Jacques Cléach, maire de Morlaix (1971-1989), le 9 mai 2003.

96. Entretien avec Jean Glavany, le 17 janvier 2005.

97. Entretien avec Jean Glavany, le 17 janvier 2005.

98. ABELES, Marc, Anthropologie de l'État, Paris, Armand Colin, 1990, p. 8. 


\section{RESUME}

La pratique des voyages de souveraineté en province est ancienne. Depuis Sadi Carnot, les présidents de la République ont réinvestit cette pratique qui apparaît à la fois comme un héritage d'Ancien Régime et comme le moment où le chef de l'État incarne l'idée républicaine. Il s'agit ici de proposer une analyse de deux visites présidentielles de François Mitterrand dans le Finistère. Celle de juillet 1981 le montre en chef des Armées dans le contexte particulier de l'alternance politique. Celle d'octobre 1985 se déroule à un moment où la cohabitation est plus que jamais probable. Comment le président Mitterrand a t-il utilisé les ressources que lui offrait le protocole pour sortir avec un surcroît de légitimité de ces deux visites présidentielles? En quoi François Mitterrand a-t-il fait de cette pratique une arme politique qu'il se présente en chef des Armées ou en Père de la nation? Cette mise en scène du pouvoir présidentiel en province reste bien, encore de nos jours, une " propagande par la vue ".

\section{ABSTRACT}

The practise of sovereign visits to the provinces is not recent. Since Sadi Carnot, the presidents of the French Republic have resumed this practise which appears both as a heritage from the Ancien Régime as well as a time when the head of the State embodies the republican idea. Here the point is to analyse a visit of François Mitterrand to Finistère. His visit in July 1981 showed him as the head of the Armies in the particular context of the changeover of political power. In October 1985, his visit happened at a time when more than ever, cohabitation appeared to be probable. How did President Mitterrand use the resources of the protocol that won him increased legitimacy from his two official visits? How did François Mitterrand change this practice into a political weapon whether he appeared as head of the Armies or as the father of the Nation? This show of the political power in the provinces is still, nowadays "a propaganda for all to see". 
